\title{
Was hilft mir das Recht auf Vergessen, wenn ich nicht für mich sein kann?
}

verfassungsblog.de/hilft-mir-das-recht-auf-vergessen-wenn-ich-nicht-fuer-mich-sein-kann/

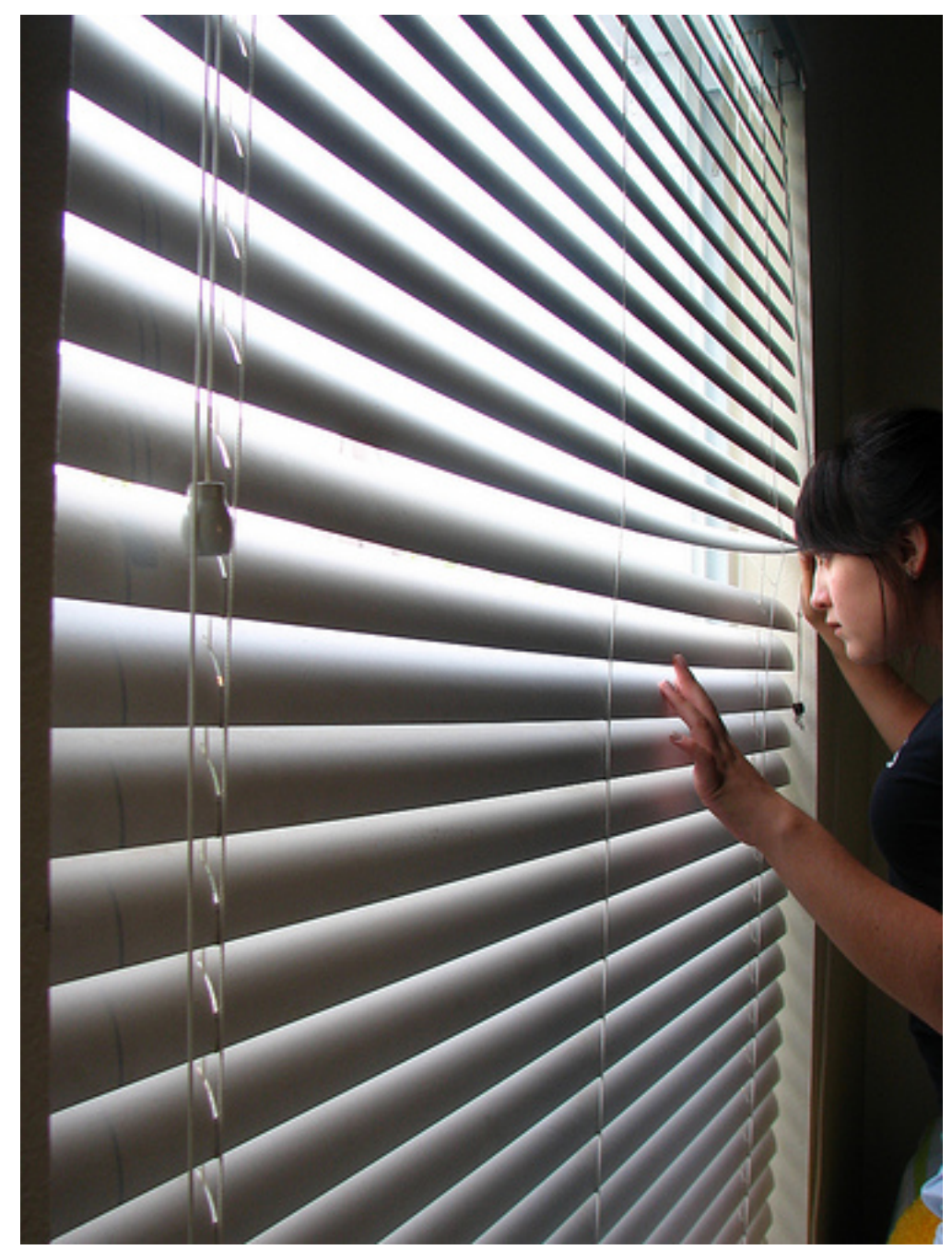

(c) hipponotized, Flickr CC BY-NC-SA 2.0

Privacy heißt, für mich bleiben zu können. Eine Grenze ziehen zu dürfen zwischen mir und der Gesellschaft, ab der sie mich in Ruhe lassen muss und ich von ihrer moralischen, politischen und ökonomischen Inanspruchnahme unbehelligt bleibe. Mich undurchsichtig machen, mich verhüllen, mich verbergen zu dürfen, in meinen Kleidern am Leib, in den Wänden meiner Wohnung, in meiner eigenen Person.

Meine Privacy zu verteidigen, dazu ist der Datenschutz da, und den Datenschutz in Europa zu verteidigen, dazu ist, wie sich vorletzte Woche herausstellte, der Europäische Gerichtshof da, der zu diesem Zweck Google verurteilt hat, Informationen über Leute nicht mehr in ihren Suchmaschinen-Ergebnissen zu listen, wenn sie das nicht wollen. Das Urteil hat eine Menge Kritik erfahren, weil es das Informationsinteresse der Öffentlichkeit und die Meinungsfreiheit derer, die Informationen verbreiten wollen, nicht ausreichend schützt. Das leuchtet mir alles durchaus ein. Aber sollten wir uns nicht mindestens genau so viel Sorgen um jenes Recht auf Privacy machen, das das Google-Urteil eigentlich schützen soll?

Letzten Dienstag war an der Humboldt-Uni als Gast von des Rule of Law Center am WZB der amerikanische Verfassungsrechtler Jack Balkin von der Yale Law School zu Besuch. Der ist nicht nur Begründer des legendären Blogs Balkinization (dazu haben wir mit inm ein Interview geführt, das wir nächste Woche hier posten werden), sondern auch einer der klügsten Köpfe der an klugen Köpfen nicht armen liberalen USVerfassungsrechtsszene. 
Balkins Thema war die Meinungsfreiheit oder genauer: Freedom of Speech im Überwachungsstaat. Es gebe, so Balkin, eine Old-School- und eine New-School-Variante der Regulierung freier Rede: Old School heißt, den Sprechenden selber ins Visier zu nehmen, inn zu knebeln, ins Gefängnis zu stecken, Strafen anzudrohen, wenn er ausspricht, was er sagen will. New School zielt dagegen auf die Infrastruktur der freien Rede - auf Telecomund Internetprovider, auf soziale Netzwerke, auf Suchmaschinenbetreiber. Nicht dem Sprechenden rücken die Behörden auf die Pelle, sondern den Betreibern der Infrastruktur: Sie werden zur Verantwortung gezogen, die freie Rede des Sprechenden nicht weiterzuverbreiten. Der Sprechende kann weiter sprechen, was er will. Es hört inn aber keiner mehr.

Dies, so Balkin, ist unendlich viel effektiver als die brutale, direkte Old-School-Regulierung. Es funktioniert im Zweifel völlig widerstandslos. Der Infrastruktur-Betreiber wird, wenn er eine solche behördliche Verfügung zugestellt bekommt, vielleicht ein bisschen brummeln. Er wird vielleicht seine Mehrkosten in Rechnung stellen. Aber wirklich wehren, mit aller Macht, die inm seine Milliarden-Bilanzsumme verleiht, wird er sich nicht. Er will schließlich nur Geld verdienen. Früher hätte der Staat, wenn er die freie Rede einschränken wollte, sich vom Spiegel bis zum Springer-Verlag lauter mächtige Player zum Feind gemacht, die an meiner Seite gekämpft hätten bis zum Umfallen. Die haben schließlich noch selber gesprochen, ihr Geschäftsmodell hing davon ab. Und heute? Google spricht ja gar nicht. Ich spreche.

Aber auch auf mich selbst, als Bürger, als Sprechenden, ist kein Verlass. Ich werde auch keinen Ärger machen. Es ist ja nicht so, dass ich nicht sprechen könnte, im Gegenteil. Es wird mir sogar unfassbar leicht gemacht. Die Behörden, und noch viel mehr die Infrastruktur-Betreiber, wünschen sich nichts dringlicher, als dass wir alle schnattern, texten, chatten, skypen, bloggen, Bilder hochladen und uns mit Katzenvideos bombardieren bis zur völligen Entkräftung, auf dass im Datenstrom der Pegelstand nicht sinkt und die unaufhörlich Konsum- bzw. Bedrohungsmuster ausspuckenden Analyse- und Filterturbinen nicht aufhören zu rotieren. Chilling effect? Aber keine Spur. Speech has never been freer easier!

Ohnehin, so Balkin, war es immer schon nur das Geschäftsmodell mächtiger Konzerne, das den Regulierungsdrang der Behörden als Gegengewicht zu bremsen imstande war: Hollywood, Medien, Riesenkonzerne mit tiefen Taschen und Heerscharen von Lobbyisten. In der Old-School-Welt liefen deren Interessen mit dem von Free Speech parallel: möglichst wenig Regulierung! Heute findet Hollywood nichts wichtiger, als Internetprovider für illegale Filmdownloads in Haftung zu nehmen. Und Amazon verdient sein Geld mit Datenanalyse, wie alle anderen Big-Data-Firmen auch. Ihr Interesse läuft nicht mit unserem parallel, sondern mit dem der Behörden.

Und wenn irgendwo noch ein großer Konzern sich für Free Speech ins Zeug wirft, dann hat er ganz anderes damit im Sinn: Dann will die Telekom damit erreichen, bei der Datendurchleitung diskriminieren zu dürfen - Free Speech heißt dann, dass niemand einem Netzbetreiber vorschreiben darf, welche Daten er transportiert und welche nicht. Dann wollen die Koch-Brüder damit erreichen, sich politischen Einfluss kaufen zu dürfen - Free Speech heißt dann, dass niemand einem reichen Mann vorschreiben darf, welchen Kongresskandidaten er unterstützt und welchen nicht. "Was Free Speech bedeutet", so Jack Balkin am Dienstag, "verändert sich unter unseren Füßen."

Das Ergebnis ist, dass der feuchte Traum der Sicherheitsbürokratie Wirklichkeit wird: Sie kann tatsächlich regulieren, was an Kommunikation in der Gesellschaft passiert. Sie hat uns im Griff. Nicht jeden einzelnen von uns, aber uns alle insgesamt dafür um so fester. Der individuelle Grundrechtseingriff ist dabei so zersplittert, kollateralisiert und aufgelöst wie der faule Immobilienkredit in einem AAA-gerateten Paket Asset Backed Securities. Weit und breit ist niemand in Sicht, dessen Protest genügend Gewicht auf die Waage bringen könnte, um diesem Traum ernsthaft im Weg zu sein. Solange wir nur unermüdlich miteinander sprechen, läuft alles wie geschmiert.

Womit wir beim Thema Privacy wären. Dass das Google-Urteil des EuGH gegen den Traum der Sicherheitsbürokratie nichts hilft, sondern im Gegenteil ihn erst richtig beflügelt, liegt auf der Hand.

Es hilft mir vielleicht, wenn jemand einen fiesen Blogpost oder ein demütigendes Foto von mir hochgeladen hat. Das ist schön. 
Aber es hilft mir nicht gegen den Befund, dass ich kaum einen Atemzug tun kann, ohne damit irgendwelche Datenturbinen anzutreiben. Es hilft mir nicht dagegen, dass ich das lidlose Auge des Überwachungsstaats auf mir ruhen fühle bei allem, was ich tue. Es hilft mir nicht gegen den Eindruck, dass man in Ruhe gelassen werden und in der Unauffälligkeit verschwinden nur noch dann kann, wenn man dauernd mittendrin ist. Es hilft mir nichts gegen die Einsicht, dass es regelrecht riskant werden kann, einfach mal die Tür hinter sich zumachen zu wollen.

Der EuGH hat ein epochales Grundsatzurteil zum Schutz meiner Privacy gefällt. Aber ich fühle mich bedrohter denn je.

\section{LICENSED UNDER CC BY NC ND}

SUGGESTED CITATION Steinbeis, Maximilian: Was hilft mir das Recht auf Vergessen, wenn ich nicht für mich sein kann?, VerfBlog, 2014/5/31, http://verfassungsblog.de/hilft-mir-das-recht-auf-vergessen-wenn-ich-nichtfuer-mich-sein-kann/. 